\title{
New pulsation analysis of the oscillating Eclipsing Binary BG Peg
}

\author{
A. Liakos \& P. Niarchos \\ National and Kapodistrian University of Athens, Faculty of Physics, \\ Department of Astrophysics, Astronomy and Mechanics, \\ GR 157 84, Zografos, Athens, Hellas
}

\begin{abstract}
New BVRI light curves of the eclipsing binary BG Peg were obtained and analysed using the Wilson-Devinney code. The observations (102 hours during a span of 108 days) were collected to perform an accurate frequency analysis of the pulsations of the primary component of the system, which is of $\delta$ Sct type. Three pulsation frequencies were identified in that star.
\end{abstract}

Accepted: July 22, 2011

Individual Objects: BG Peg

\section{Introduction}

The story of the discovery of the variability of BG Peg ( V=10.5 mag, $\mathrm{P} \sim$ 1.95 days) is not very clear and is a hidden chapter in the history of variable star research. Prager \& Shapley (1941) listed this system as a variable and reported that the discovery was made by Zessewitsch (1931) in Moscow, but the exact reference is missing from the literature. Budding (1984) found the mass ratio of the binary system to be 0.51, Brancewicz \& Dworak (1980) and Svechnikov \& Kuznetsova (1990) calculated the absolute parameters of the system's components and found different mass values. However, in these three works the system is classified as A2 type. Independently, and almost simultaneously, Soydugan et al. (2009) and Dvorak (2009) announced the pulsating nature of the primary component of the system and they found a dominant pulsating frequency of $\sim 25.5 \mathrm{c} / \mathrm{d}$. Most recently, a comprehensive study of this eclipsing binary (EB) was made by Soydugan et al. (2011), with frequency analyses based on $B$ and $V$ photometry. In the same work, an orbital 
period study was also performed, resulting in evidence for a tertiary companion, mass loss from the system and mass transfer between the components.

In the study presented here, we present and analyse four-colour light curves covering a three-month time span, to identify pulsation frequencies in the data. Our analysis assumes a third light contribution to the system's apparent brightness.

\section{Observations and data reduction}

The BVRI photometric observations were carried out during 15 nights from 6 August to 21 November 2010 at Gerostathopoulion Observatory of the University of Athens. Two instrumentation set-ups (a 40-cm Cassegrain and a 25- $\mathrm{cm}$ Newtonian Reflector telescopes equipped with the ST-10 XME and the ST8 XMEI CCD cameras, respectively) were used to collect the data. Aperture photometry techniques were applied to CCD images and differential magnitudes were obtained with the software package MuniWin v.1.1.26 (Hroch 1998). TYC 1698-1148-1 ( $\mathrm{V}=10.4 \mathrm{mag})$ and TYC 1698-1078-1 ( $\mathrm{V}=11.7 \mathrm{mag})$ were used as comparison and check stars, respectively. The mean standard deviation of the data points were $5.8,6.1,7.3$ and $8.6 \mathrm{mmag}$ for $\mathrm{B}, \mathrm{V}, \mathrm{R}$ and I filters, respectively.

\section{Light curve analysis}

The light curves of the BG Peg system were analysed simultaneously using the PHOEBE v.0.29d software (Prša \& Zwitter 2005) which uses the method of the 2003 version of the Wilson-Devinney (WD) code (Wilson \& Devinney 1971; Wilson 1979, 1990). The 'Multiple Subsets' procedure was used to approach our final solution. Lacking a spectroscopic mass ratio, we applied the ' $q$-search' method with a step of 0.1 , with trials in Mode 2 (detached system) and Mode 5 (semidetached system with the secondary component filling its Roche Lobe), to find plausible 'photometric' estimates for the mass ratio $q_{p h}$. Based on the secondary component's potential, which fits better to a star which fills its respective Roche Lobe (cf. Ibanoğlu 2006), Mode 5 was selected as the better way to describe the geometry of this system, and we find $q_{p h} \sim 0.2$. In the subsequent analysis, this value was set as initial input and treated as free parameter. The temperature of the primary was assigned a value of $9000 \mathrm{~K}$ according to its spectral type by using the correlation given in the tables of Cox (2000) and was fixed during the analysis. The temperature of the secondary $T_{2}$ was adjusted. The albedos, $A_{1}$ and $A_{2}$, and gravity darkening coefficients, $g_{1}$ and $g_{2}$, were set to generally adopted values (von Zeipel 1924; Lucy 1967; Rucinski 1969) for the given spectral types of the components. The linear limb 
Table 1: Results of light curves solution with the corresponding errors given in parentheses.

\begin{tabular}{|c|c|c|c|c|c|c|c|}
\hline Parameter & \multicolumn{2}{|c|}{ System related } & \multicolumn{5}{|c|}{ filter depended } \\
\hline i [deg] & & $(1)$ & & $B$ & $\mathrm{~V}$ & $\mathrm{R}$ & $\mathrm{I}$ \\
\hline \multirow[t]{3}{*}{$\mathrm{q}\left(=m_{2} / m_{1}\right)$} & & (1) & $\mathrm{L}_{1} / \mathrm{L}_{T}$ & $0.955(1)$ & $0.927(2)$ & $0.903(2)$ & $0.873(3)$ \\
\hline & Compor & it related & $\mathrm{L}_{1} / \mathrm{L}_{T}$ & $0.045(1)$ & $0.073(1)$ & $0.097(1)$ & $0.127(1)$ \\
\hline & $\mathrm{P}$ & $\mathrm{S}$ & $\mathrm{x}_{1}$ & 0.536 & 0.460 & 0.380 & 0.300 \\
\hline $\mathrm{T}[\mathrm{K}]$ & $9000^{a}$ & $5095(12)$ & $x_{2}$ & 0.849 & 0.706 & 0.609 & 0.512 \\
\hline$\Omega$ & $3.67(1)$ & 2.28 & \multicolumn{5}{|c|}{ fractional radii } \\
\hline$g^{a}$ & 1 & 0.32 & & pole & point & side & back \\
\hline $\mathrm{A}^{a}$ & 1 & 0.5 & $r_{1}$ & 0.289 & 0.297 & 0.293 & 0.295 \\
\hline$\sum r e s^{2}$ & & & $r_{2}$ & 0.240 & 0.500 & 0.249 & 0.281 \\
\hline
\end{tabular}

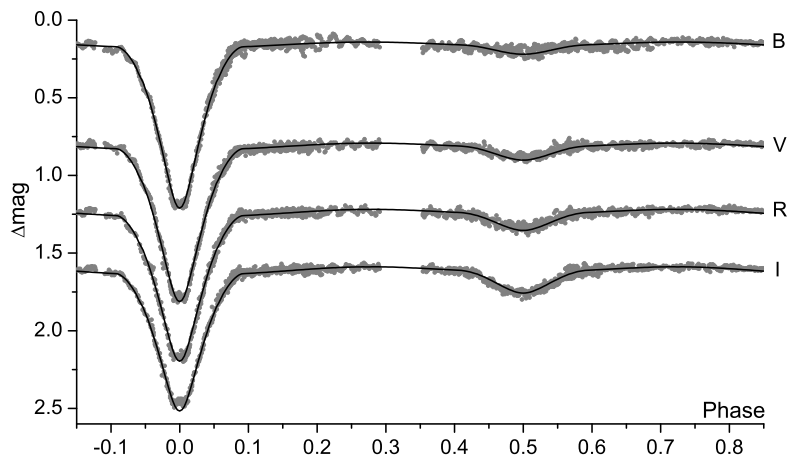

Figure 1: Synthetic (solid lines) and observed (grey points) light curves of BG Peg.

darkening coefficients, $x_{1}$ and $x_{2}$, were taken from the tables of van Hamme (1993); the dimensionless potentials $\Omega_{1}$ and $\Omega_{2}$, the fractional luminosity of the primary component $L_{1}$ and the inclination $i$ of the system's orbit were set in the programme as adjustable parameters. Due to a possible existence of a tertiary component orbiting the EB (Soydugan et al. 2011), the third light option $I_{3}$ was applied, but it resulted in unrealistic values. As a result, it is not included in the final solution. Synthetic and observed light curves are presented in Fig. 1, with the corresponding parameters of the model fits given in Table 1.

\section{Frequency analysis}

A frequency search of the light curve residuals, except near the primary eclipse, was performed in the frequency range $3-80 \mathrm{c} / \mathrm{d}$, which is a typical range for 
Table 2: The frequency analysis results of BG Peg.

\begin{tabular}{|c|c|c|c|c|c|c|c|c|}
\hline & $\begin{array}{c}\mathbf{F} \\
{[\mathrm{c} / \mathrm{d}]}\end{array}$ & $\begin{array}{c}\mathbf{A} \\
{[\mathrm{mmag}]}\end{array}$ & $\begin{array}{c}\Phi \\
{[\mathrm{deg}]}\end{array}$ & $\mathrm{S} / \mathrm{N}$ & $\begin{array}{c}\mathbf{F} \\
{[\mathrm{c} / \mathrm{d}]}\end{array}$ & $\begin{array}{c}\mathbf{A} \\
{[\mathrm{mmag}]}\end{array}$ & $\begin{array}{c}\phi \\
{[\mathrm{deg}]}\end{array}$ & $\mathrm{S} / \mathrm{N}$ \\
\hline & \multicolumn{4}{|c|}{$B$ - filter } & \multicolumn{4}{|c|}{$V$ - filter } \\
\hline$f_{1}$ & $25.544(1)$ & $13.1(6)$ & $8(3)$ & 14.8 & $25.544(1)$ & $11.7(6)$ & $10(3)$ & 18.7 \\
\hline$f_{2}$ & 23.085 (1) & $8.4(6)$ & $117(4)$ & 9.9 & $23.085(1)$ & $6.3(6)$ & $118(5)$ & 10.4 \\
\hline$f_{3}$ & $22.061(1)$ & $6.1(6)$ & $19(6)$ & 6.5 & $22.061(1)$ & $6.1(6)$ & $8(5)$ & 9.0 \\
\hline$\sum r e s^{2}$ & \multicolumn{4}{|c|}{0.017} & \multicolumn{4}{|c|}{0.014} \\
\hline & \multicolumn{4}{|c|}{$R$ - filter } & \multicolumn{4}{|c|}{ I- filter } \\
\hline$f_{1}$ & $25.544(1)$ & $8.3(6)$ & $359(4)$ & 9.8 & $25.543(1)$ & $7.1(6)$ & $16(5)$ & 9.8 \\
\hline$f_{2}$ & $23.094(1)$ & $4.3(6)$ & $147(8)$ & 4.7 & 23.085 (1) & $4.0(6)$ & $111(8)$ & 5.4 \\
\hline$f_{3}$ & $22.062(1)$ & $3.9(6)$ & $9(9)$ & 4.1 & $22.060(1)$ & $3.3(6)$ & $19(10)$ & 4.7 \\
\hline$\sum r e s^{2}$ & \multicolumn{4}{|c|}{0.015} & \multicolumn{4}{|c|}{0.015} \\
\hline
\end{tabular}

$\delta$ Scuti stars (Breger 2000; Soydugan et al. 2006). We used the software package PERIOD04 v.1.2 (Lenz \& Breger 2005), which is based on the classical Fourier analysis. After each frequency identification, the residuals were subsequently pre-whitened for the next one. The frequency search stopped when the detected frequency(ies) had a signal-to-noise ratio $(\mathrm{S} / \mathrm{N})$ less than 4 and an amplitude value lower than the significance limit ( $4 \sigma$ level). An additional frequency search was made in the range $0-3 \mathrm{c} / \mathrm{d}$, for frequencies which potentially could be caused by terrestrial atmospheric effects, instrumental drifts or imperfect light curve fits. Low frequencies can also result from $g$-mode pulsations (Breger 2005), which are expected to be traced in the data of more than one filter. In this data set, frequencies with values less than $3 \mathrm{c} / \mathrm{d}$ were indeed detected, but they were found to be different for each filter. Thus, they cannot be considered as physically intrinsic to the BG Peg system and they are excluded from the final solution shown in Table 2.

The amplitude spectrum, the spectral window and the Fourier fit on the longest data sets are plotted in Fig. 2.

\section{Discussion and conclusions}

We have reduced and analysed time series BVRI photometry of BG Peg. BG Peg was found to be a semidetached system with its cooler and less massive component filling its Roche Lobe. Therefore, the eclipsing pair, according to the definition suggested by Mkrtichian et al. (2004), is a classical oscillating Algol (oEA). A third light contribution was included in the analysis, due to a suggested existence of a tertiary component (Soydugan et al. 2011), but the results did not support it. Our light curves solution, however, is in very good agreement with that of Soydugan et al. (2011). 


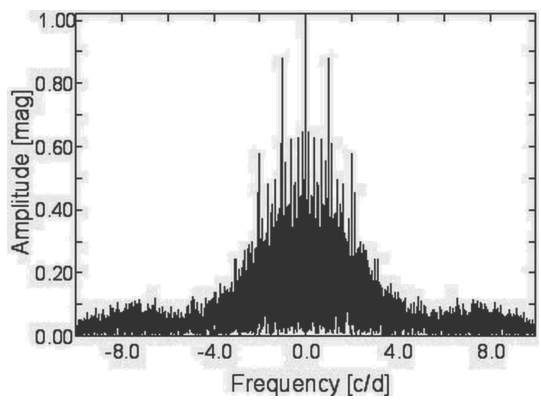

(a)

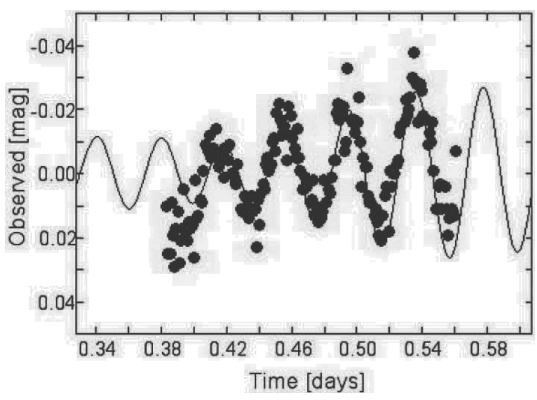

(b)

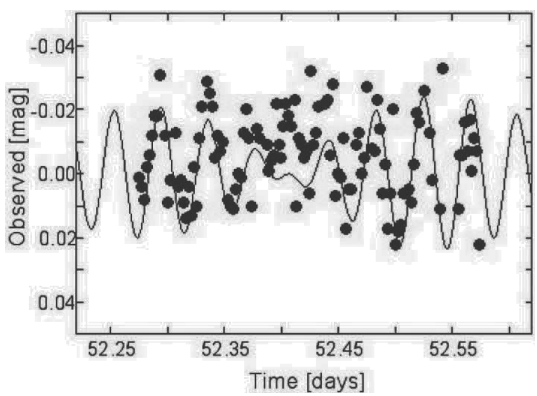

(c)

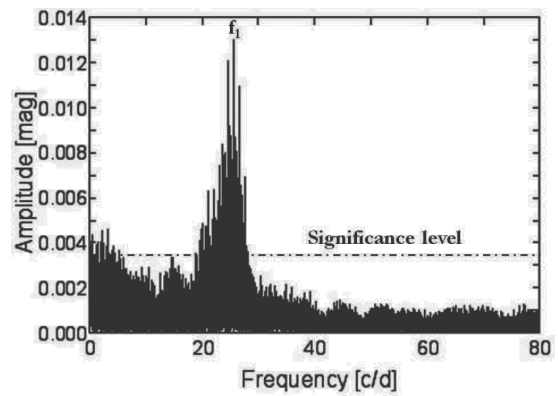

(d)

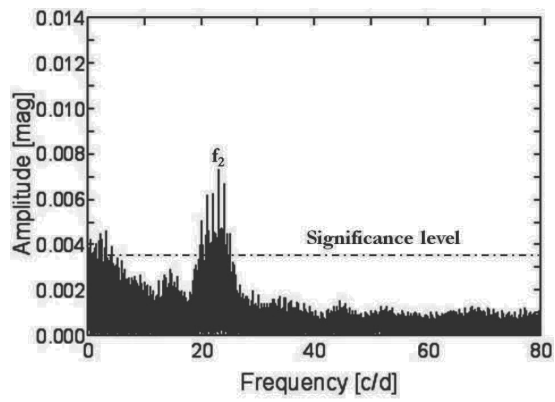

(e)

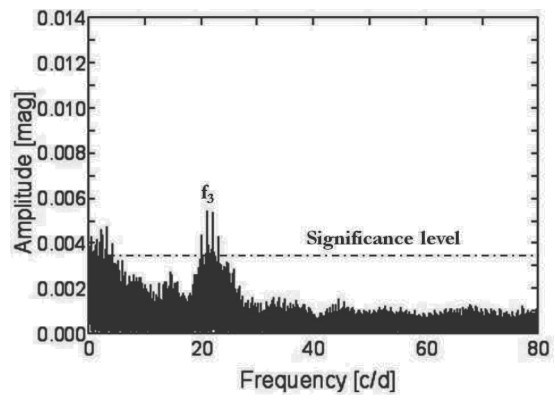

(f)

Figure 2: (a): The spectral window plot, (b)-(c) the Fourier fit on the longest data sets, and (d)-(f) the Fourier Amplitude spectrum where the detected frequencies and the significance level are indicated. 
Three significant pulsation frequencies were detected in all bandpasses with decreasing amplitudes from B to I. Soydugan et al. (2011) found only two frequencies for the hotter component of BG Peg. We confirm the fundamental pulsation frequency $f_{1}$ reported by Soydugan et al. (2011), but we cannot confirm the second frequency in their study $\left(f_{2} \sim 21.06 \mathrm{c} / \mathrm{d}\right)$. The two new frequencies we have found might be because of our better time coverage: 102 hrs on 15 nights during 108 days for our study, versus $\sim 56$ hrs on 13 nights during 87 days for the Soydugan et al. (2011) study.

Radial velocity measurements are certainly needed to derive the spectroscopic mass ratio and for a more coherent study of the primary component's pulsational characteristics.

Acknowledgments. This work has been financially supported by the Special Account for Research Grants No 70/4/9709 of the National \& Kapodistrian University of Athens, Hellas. We used the SIMBAD database, operated at CDS, Strasbourg, France, and the Astrophysics Data System Bibliographic Services (NASA). We thank the anonymous referee for his valuable comments, which improved the quality of the paper.

\section{References}

Brancewicz, H.K. \& Dworak, T.Z. 1980, AcA, 30, 501

Breger, M. 2000, ASPC, 210, 3

Breger, M. 2005, ASPCS, 333, 138

Budding, E. 1984, Bull. D'Inf. Cent. Donnees Stellaires, 27, 91

Cox, A.N. 2000, Allen's Astrophysical Quantities $4^{\text {th }}$ ed., Springer-AIP press

Dvorak, S. 2009, CoAst, 160, 64

Hroch, F. 1998, Proceedings of the 29th Conference on Variable Star Research, 30 Ibanoğlu, C., Soydugan, F., Soydugan, E., Dervișoğlu, A., 2006, MNRAS, 373, 435 Lenz, P. \& Breger, M. 2005, CoAst, 146, 53

Lucy, L.B. 1967, Zeitschrift für Astrophysik, 65, 89

Mkrtichian, D.E., Kusakin, A.V., Rodriguez, E., et al. 2004, A\&A, 419, 1015

Prager, R. \& Shapley, H. 1941, AnHar, 111, 1

Prša, A. \& Zwitter, T. 2005, ApJ, 628, 426

Rucinski, S.M. 1969, AcA, 19, 245

Soydugan, E., Soydugan, F., Demircan, O., Ibanoğlu, C. 2006, MNRAS, 370, 2013

Soydugan, E., Soydugan, F., Şenyüz, T., et al. 2009, IBVS, No 5902

Soydugan, E., Soydugan, F., Șenyüz, T., Püsküllü, Ç., Demircan, O. 2011, NewA, 16,72 
Svechnikov, M.A. \& Kuznetsova, E.F. 1990, Catalogue of Approximate Photometric and Absolute Elements of Eclipsing Variable Stars, A.M. Gorky University of the Urals, Sverdlovsk

van Hamme, W. 1993, AJ, 106, 2096

von Zeipel, H. 1924, MNRAS, 84, 665

Wilson, R.E. \& Devinney, E.J. 1971, ApJ, 166, 605

Wilson, R.E. 1979, ApJ, 234, 1054

Wilson, R.E. 1990, ApJ, 356, 613 
